\title{
ON-CHIP ABSORPTION AND FLUORESCENCE SPECTROSCOPY WITH POLYDIMETHYLSILOXANE (PDMS) MICROFLUIDIC FLOW CHANNELS
}

\author{
Mark L. Adams, Stephen Quake, and Axel Scherer \\ Electrical Engineering and Applied Physics, California Institute of Technology, CA, U.S.A.
}

\begin{abstract}
We describe a system, which integrates PDMS microfluidic flow channels, a CMOS Active Pixel Sensor (APS) imager, and commercial light sources for performing visible absorption and fluorescence spectroscopy. PDMS elastomer allows for spectroscopic measurements to be performed in the visible ( 350 to $750 \mathrm{~nm}$ ) wavelength range. Along with a sensitive silicon photodetector such as an APS imager, measurements can be made on low concentrations of solutions by placing the microfluidic flow channels directly on the imager. Emission sources for absorption measurements are typically commercial LEDs with peak emission wavelengths that match the peak absorption wavelengths of the material under test. For calibration purposes, known dyes are tested and compared with the results obtained from the imager. Fluorescence measurements are performed in much the same way, but a band-blocking filter is placed between the microfluidic channels and the imager to greatly attenuate the pump wavelength. Typical emission sources for fluorescence are commercial Ar ion lasers and semiconductor lasers. We feel that the system has great advantages due to its miniaturized size and small fluid volumes required. Further system miniaturization is also possible due to the integration of emission sources and the expandability of CMOS imagers to integrate on board image processing.
\end{abstract}

Keywords: Micro-fluidics, Spectrometers, Hyper-spectral imaging

\section{INTRODUCTION}

Over the past few years, the use of replication molding for the definition of microfluidic systems in elastomers has allowed the rapid development of compact analysis systems useful for chemical sensing and biological diagnostics. For example, fluorescently activated cell sorters (17) based on pumps, valves and channels defined in RTV silicone elastomers have demonstrated excellent throughput and sorting accuracy. These have been fabricated inexpensively into very small and robust micro-fluidic devices. Chemical surface pretreatment of specific areas within a flow channel has led to the possibility of developing very compact disease diagnostic chips, and even single molecule sizing systems can be built from elastomeric flow channels (21). In all of these applications, the overall size of the analysis system is typically limited by the dimensions of the optical excitation and detection components, and miniaturization of the readout optics is therefore very desirable. However, miniaturization of grating-based spectrometer geometries ultimately is limited by a reduction of the spectral resolution, which can be predicted from the optical pathlengths between the grating and the detection slit. For example, multi-wavelength $4 \mathrm{mmx} 12 \mathrm{~mm}$ spectrometers operating at $1500 \mathrm{~nm}$ typically yield a measured spectral resolution of approximately $1 \mathrm{~nm}$. This compromise between resolution, insertion losses and size has in the past limited the minimum size of such optical analysis systems. Much better spectral performance can be obtained by using dielectric filters, which can be directly deposited onto detector arrays to form multi-wavelength detector arrays. Such filtering has in the past been used for monolithic hyper-spectral imaging applications. Filtered detector arrays offer an inherent opportunity for the miniaturization of spectroscopic instruments in microfluidic applications, with the additional opportunity of obtaining low-resolution "lens less" images of the contents in the flowchannel.

One of the most important advantages of using elastomeric flow channels is the inherent transparency of the elastomer material in the visible wavelength range. Many semiconductor based microfluidic structures previously proposed have suffered from the inability to perform optical analysis of the device's contents in the visible and near-UV spectral ranges. Due to the absorption edge of silicon, for example, optical measurements in flow channels defined by this material are typically limited to the infrared range and visible/UV spectroscopy is virtually impossible to perform without using very elaborate geometries. For applications such as biochemistry, this poses a severe limitation since many absorption and fluorescence experiments are based on visible/UV fluorescent dyes. Silicone elastomers circumvent this problem since they are optically transparent and have similar UV absorption characteristics to those of glass. This property enables the easy integration of elastomer microfluidic devices with standard optoelectronic sources and detectors. Moreover, silicone elastomers are simple to integrate on top of already fully fabricated detector arrays, forming a hermetic seal to the passivation layer of the detector arrays.

Miniaturization of absorption spectrometers will advance rapidly over the next few years, partly due to development of short wavelength LED's and faster computer 


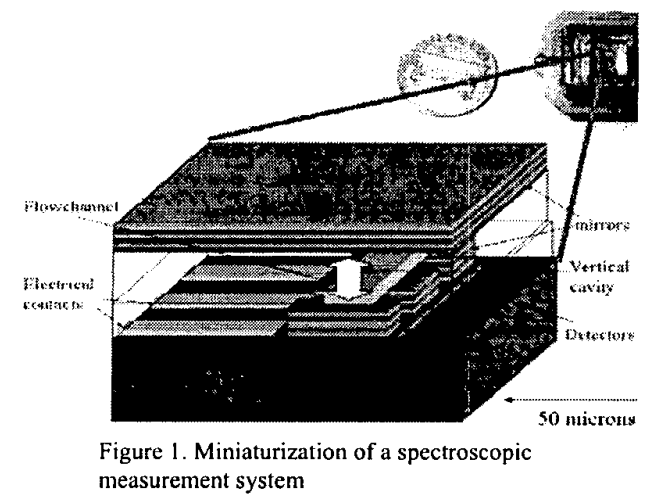

interconnects, as well as the development of inexpensive and high-quality CMOS imaging arrays. In thisp aper, we describe the performance of a compact and inexpensive spectrometer geometry with spectrally filtered detector arrays underneath elastomeric microfluidic channels.

\section{EXPERIMENTAL.}

\section{A. Replication Molding}

Replication molding, sometimes also referred to as soft lithography, dates to prehistoric times, and has been used to make phonographic records, blazed grating optics (13), and stamps for chemical patterning (14). Through the use of replication molding techniques, we have in the past demonstrated many microfluidic devices such as peristaltic pumps, pneumatic valves, circular mixers, and fluorescently activated cell sorters (15-20). Replication molding involves curing an elastomer, typically silicone based, on a microscopic mold. The mold can be generated through bulk or surface micromachining, but is typically generated via photolithography. The simplest method for creating a mold suitable for a microfluidic system consists of exposing thick UV sensitive photoresist using high-resolution contact photolithography. The resulting mold pattern is then developed and treated with a delamination agent to prevent adhesion between the mold and the cured elastomer. The elastomer can be cast on top of the mold by either pouring a thick layer, or spinning a thin layer onto the patterned surface. To define simple pneumatic valves with picoliter dead volumes, multilayer replication techniques are used, in

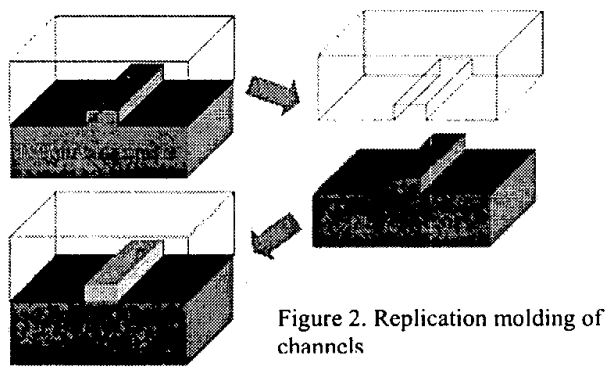

which a pneumatic layer is aligned onto the fluidic layer. The elastomer between the two layers is usually thinner than 10 microns, and can easily be deflected with low pressures introduced from the pneumatic control source. Three of these valves can be integrated to define a peristaltic pump, to move solution over the filtered detectors, which can provide spectroscopic information about the analyte flowing in the channel. Furthermore, valves and pumps can be combined to define fluidic cell sorters with feed-back in which the cell examination time can be dynamically controlled during the sorting process.

\section{B. CMOS Absorption Spectroscopy}

The external optical excitation and read-out equipment setup used in the experiments described here include a $588 \mathrm{~nm}$ light emitting diode and a CMOS camera chip. One key difference between our measurement system and more conventional cell-sorting systems is that no lenses were used for imaging. We find that a very simple approach to reducing the size of a spectroscopic imaging system is to eliminate the need for focusing optics by placing the microfluidic devices directly onto the imaging detector array. This detector can consist of silicon based avalanche photodiodes (APDs), charge coupledd evices (CCDs), or CMOS integrated $\mathrm{p}-\mathrm{n}$ diode sensors. All of these devices are commercially available at reasonable costs. CCD arrays, although in general more sensitive, suffer from the need to read out the entire image information in order to determine intensity information from the pixels underneath the flowchannels. Avalanche photodiodes typically require larger areas, and thus significantly reduce the resolution of the imaging system. CMOS imagers, on the other hand, offer direct control over individual pixels, and, since most of the area of the image array is typically not used, can provide much faster response times and long integration times. Although the lateral resolution of these imagers cannot match that of an optical microscope, it is suitable for most visible spectroscopy experiments on larger objects. The highest resolution of such a proximity imaging system is determined by the pixel size on the imaging array, and can be less than 10 microns. The sensitivity of the imaging system is in turn dependent on the active area of the pixel, as well as leakage currents in the pixels. Other factors that determine the performance of an imaging detector array in a spectrometer application are its sensitivity and dynamic range. The sensitivity becomes extremely important when examining picoliter volumes with a correspondingly small optical interaction length. Here we have chosen a CMOS imaging array as the sensor of our spectroscopic measurement system. This choice was based on the ease of directly addressing individual pixels in the array, and the opportunity for changing the integration time per pixel for more sensitive analysis. Figure 3 shows the geometry used for our spectroscopic measurements. The light source was a 588 AlInGaP light emitting diode, placed above the flow channels, which in turn were directly placed on the image array. 


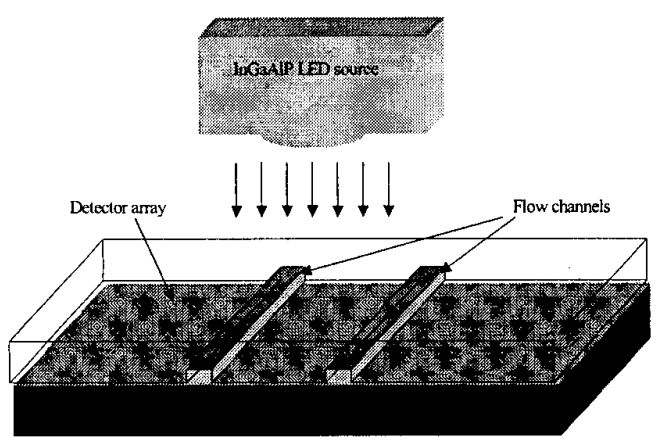

Figure 3. Absorption measurement system

Since the typical size of an elastomer microfluidic channel is on the order of 50 to 250 microns wide by 10 to 20 microns deep, the absorption path length is quite small compared to more conventional cuvette-based absorption spectrometers with interaction lengths $100-1000$ times larger. According to the Beer-Lambert law, the absorbance $A$ is proportional to the concentration of the absorbing material $c$ and the absorption pathlength $l$, so that

$$
A=\epsilon c l
$$

where $\epsilon$ is the molar absorption constant or molar absorptivity. Thus, the difference in the expected detected intensity of a channel filled with reagent versus a channel filled with water is very small for dilute solutions. Therefore the higher the sensitivity of the detectors in the sensor array, the greater the concentration range that can be detected.

Our first absorption experiment was performed using a tenbit resolution black and white CMOS imager provided by NASA's Jet Propulsion Laboratory. This imager has a typical pixel size of $12 \mathrm{um}$, a dynamic range $>65 \mathrm{~dB}$, and a responsivity $>1.3 \mu \mathrm{V} /$ photon at room temperature. The active imaging area consisted of $512 \times 512$ pixels. First, we determined the minimum concentration of dye, which can be detected in this system. We tested the absorptivity of various concentrations of bromophenol blue (Aldrich Chemical Company, Inc. \#62625-28-9) on a calibrated Shimadzu BioSpec 1601 spectrophotometer with solution filled into $1 \mathrm{~cm}$ cuvettes. The molar absorption constant was then calculated and a curve fit was applied to generate the control data for a 14um channel. Next, a polydimethylsiloxane (PDMS) microfluidic chip consisting of eleven 100um wide by 14 um deep channels spaced 100 um apart was placed directly on our CMOS imaging chip. Two channels were filled with each concentration of interest and one channel was filled with water for background measurements. Figure 3 shows a typical image of light transmission through the multi-channel silicone structure observed by the CMOS imager. The illumination

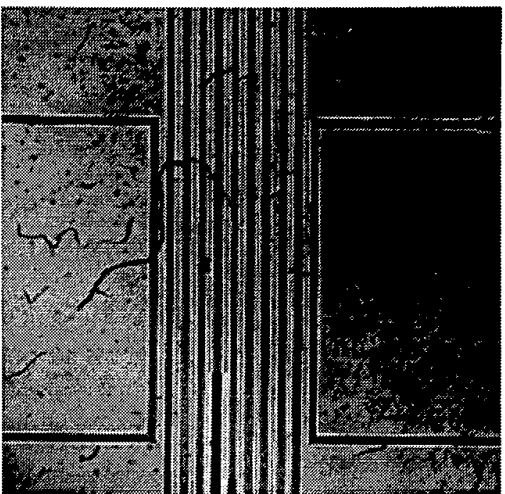

Figure 4. A PDMS microfluidic chip imaged by the JPL APS CMOS imager. The light source is a $588 \mathrm{~nm}$ Imax AlInGaP LED and the channels contain bromophenol blue solutions. The dark channel areas are where air entered the channel.

source consisted of a Yellow AlInGaP LED with $\lambda_{\max }=588 \mathrm{~nm}$ and $\mathrm{I}_{0}=1500 \mathrm{mcd}$, and was optimized for the absorption peak of bromophenol blue. The results of the experiment are summarized in Figure 5. A similar test was conducted on Orange G, excited with light at $470 \mathrm{~nm}$. From these figures, it is seen that the monolithic CMOS device displays a comparable sensitivity to the commercial Shimadzu spectrometer system.
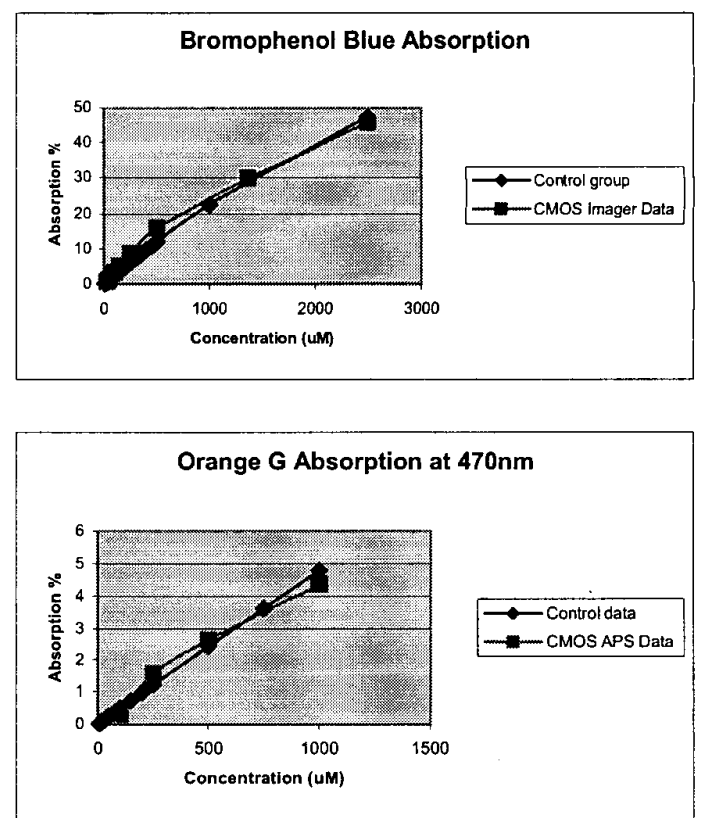

Figure 5. Absorption spectra of various Bromophenol Blue and Orange $G$ concentrations taken with a Shimadzu spectrophotometer and the APS CMOS imager.

The important advantages of the imager spectrometers over more conventional absorptance spectroscopy systems include the capability to characterize spectra from picoliter 


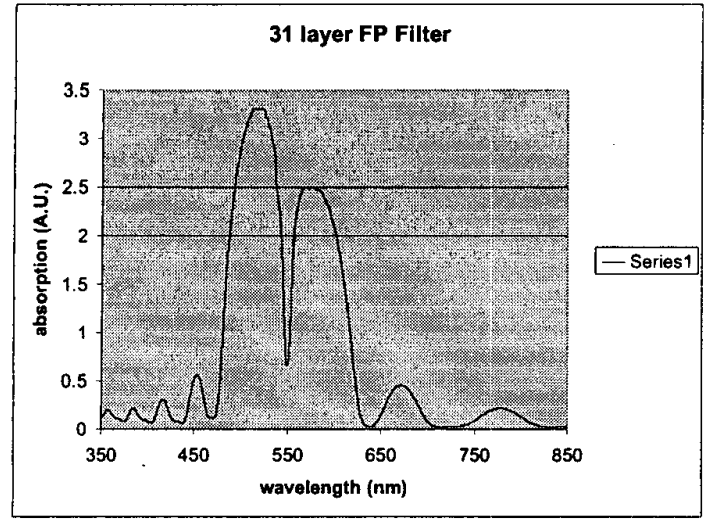

Figure 6. Absorption spectra of a 31 layer $\mathrm{SiO}_{2} / \mathrm{Si}_{3} \mathrm{~N}_{4} \mathrm{Fabry}$ Perot Cavity

volumes, and enable the observation of many channels in parallel. Individual detectors can be filtered by using $\mathrm{Si}_{3} \mathrm{~N}_{4} / \mathrm{SiO}_{2}$ multilayer Fabry-Perot cavities (Figure 6) deposited onto the silicon detector array before definition of the fluidic structures. Filters can be deposited with a deliberate thickness variation in order to obtain a specific wavelength response for each detector in the imaging array, providing a hyper-spectral imaging array. By pumping the solution of interest over these filtered detectors and observing their response, a spectrum of the absorption or fluorescence of a very small solution volume can be obtained.

\section{Fluorescence Spectroscopy}

During fluorescence spectroscopy, the sample under test is excited with a light source whose wavelength is close, within $40-80 \mathrm{~nm}$, to the emitted fluorescent light. Typically the pump source is much brighter than the fluorescence signal, especially for experiments involving small numbers of fluorescing dye molecules, such as when performing

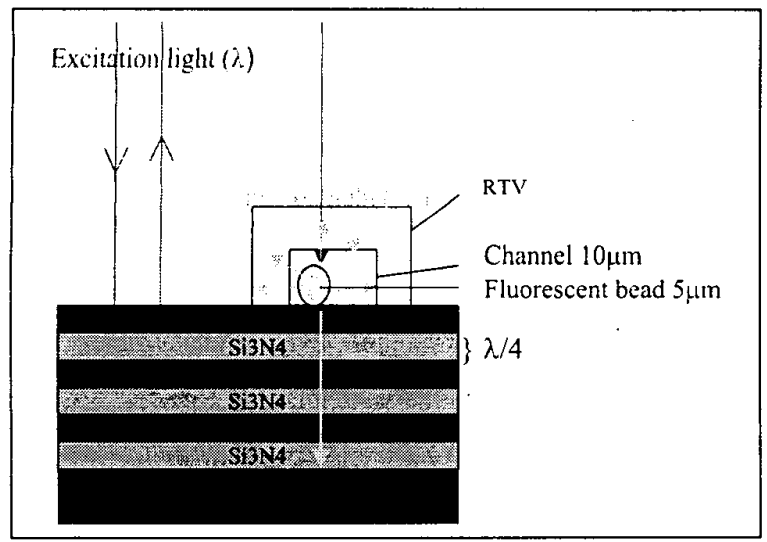

Figure 7. A quarter wavelength dielectric thin film filter grown on the CMOS imager to block the excitation light, but pass, with minimal loss, the emission light. single cell detection. Without a very efficient filter, the pump beam saturates the imager, precluding any chance of identifying the fluorescent signal. The blocking filter, which is tuned to the pump wavelength, must be placed between the microfluidic device and the imager, and should be transparent at the fluorescent wavelength. The filter can

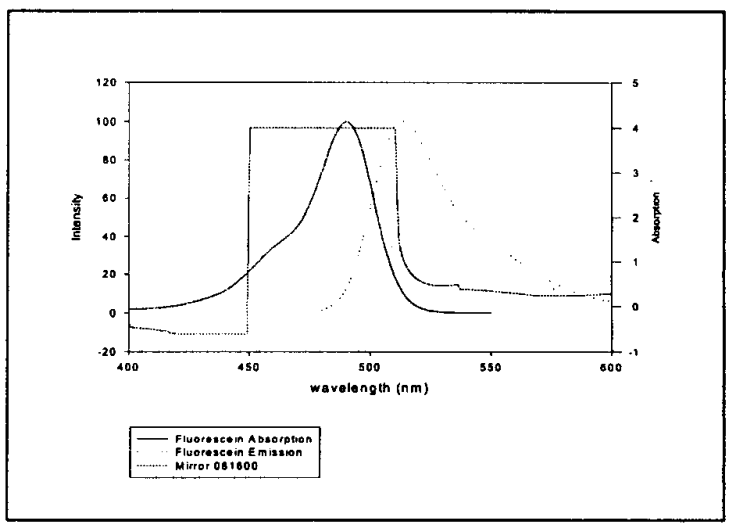

Figure 8. Fluorescent absorption and emission spectra for fluorescein. Blocking filter absorption spectrum shown in relation to second $y$ axis. The emission peak corresponds to a region just outside the block band where the transmission is roughly $50 \%$. This can easily be improved by constructing a narrower band-blocking filter.

easily be fabricated as a carefully grown dielectric thin-film mirror (Figure 7). A typical filter, deposited by reactive sputter deposition of alternate $\lambda / 4$ layers of silicon dioxide and silicon nitride, is transparent at the fluorescent wavelength, and blocks over $99 \%$ of the incident pump wavelength. We use diluted fluorescein dye to test the performance of our monolithic fluorescence system. Figure 8 shows the absorption and emission spectra of the fluorescein dye, together with the reflectivity spectrum of the dielectric blocking mirror. Since the mirrors and flow channel are directly deposited onto the silicon CMOS detector array, we can use the lens less contact image from this array to differentiate between concentrations of fluorescein. Spectrally resolved fluorescence measurements are also possible by slowly varying the spectral position of the reflectivity edge of the dielectric blocking mirror and measuring fluorescence intensities in different sensor pixels protected with filters with different reflectivity edges. The requirement for obtaining a high quality fluorescence image on a miniaturized chip-based spectrometer relics on very efficient blocking of the incident excitation light, which would otherwise overwhelm the fluorescing signal. Figure 9 depicts a sample image acquired with our system.

\section{Emission Sources}

We have demonstrated the monolithic integration of the microfluidic device directly on an image sensor array. The next component of the system, which must be miniaturized, is the light source. In a typical visible spectrophotometer 


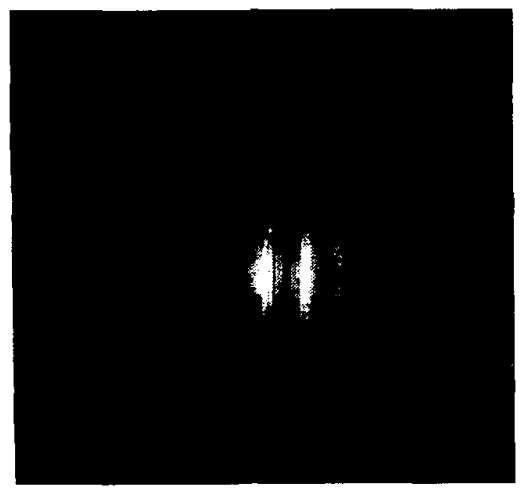

Figure 9. $170 \mathrm{mM}$ and $85 \mathrm{mM}$ fluorescein in $100 \mu \mathrm{m}$ wide by $14 \mu \mathrm{m}$ flow channels on $460 \mathrm{~nm}$ blocking filter illuminated by Ar ion laser at 488 with no. 2 and no. .5 ND filters in line

the light source is generally a tungsten or tungsten-iodine filament lamp with some models also including light emitting diodes. In a miniature spectrometer, the appropriate light source depends heavily upon the application and heat dissipation problems. The most convenient alternatives include solid-state light emitting diodes, laser diodes, white light sources, and perhaps even the sun. For infrared analysis, the source might also be a tungsten filament lamp with-a specific color filter placed directly over the microfluidic device. Although for many applications, an array of vertical cavity surface emitting lasers (VCSELs) could be desirable, such laser sources are very difficult to construct in the most interesting UV/visible wavelength range. Instead of using lasers, high finesse optical cavity filters can be defined on top of LED arrays to obtain filtered light sources, which can be directly placed on top of the microfluidic channel, which in turn is placed on top of a detector array, to create a fully functional on-chip visible spectrometer.

\section{CONCLUSION}

An ideal microfluidic analysis system would consist of inexpensive and disposable fluidic components, and a very compact, robust and monolithic optical excitation and measurement system. The optical read-out system could remain reusable for many experiments, but should be very sensitive and provide diagnostic imaging information on the condition of the flow channels. With the appropriate information processing, such an integrated system could yield rapid, accurate results in a very short time. This coupled with the flexibility of soft lithography shows much promise toward the definition of a spectroscopic laboratory on a chip in which either absorption or fluorescence can be measured. Here, we have described one such geometry, which satisfies some of the requirements of an integrated measurement system, in which intelligent analysis of microscopic fluid volumescan be undertaken. We believe that this approach, when optimized sensor arrays are used, can offer equivalent performance to commercially available spectrophotometers with the opportunity of optically monitoring many fluidic channels the same time.

\section{ACKNOWLEDGMENTS}

We would like to thank the Department of Defense Advanced Research Projects Agency (DARPA) and the National Science Foundation (NSF) for their support of this research. We would also like to thank NASA's Jet Propulsion Laboratory and the Advanced Imaging group for providing the APS CMOS imager.

\section{REFERENCES}

1. L.M. Roylance and J.B. Angell, IEEE Trans. Electron. Devices ED-26, 1911 (1979).

2. N. Yazdi, F. Ayazi, K. Najafi, Proc. IEEE 86, 1640 (1998).

3. O.N. Tufte, P.W. Chapman, D. Long, J. Appl Phys. 33, 3322 (1962).

4. L. Kuhn, E. Bassous, R. Lane, IEEE Trans. Electron. Devices ED-25, 1257 (1978).

5. L.Y. Lin, E.L. Goldstein, R.W. Tkach, IEEE J. Selected Top. Quantum Electron. 5, 4 (1999).

6. R.S. Muller and K.Y. Lau, Proc. IEEE 86, 1705 (1998).

7. L.J. Hornbeck and W.E. Nelson, OSA Tech Dig. Ser. 8, 107 (1988).

8. D.J. Harrison et al., Science 261, 895 (1993)

9. S.C. Jacobson, R. Hergenroder, L.B. Koutny, J.M. Ramsey, Anal. Chem. 66, 1114 (1994).

10. M.U. Kopp, A.J. de Mello, A. Manz, Science 280, 1046 (1998)

11. S. Shoji, Top. Curr. Chem. 194, 163 (1998).

12. P. Gravesen, J. Branebjerg, O.S. Jensen, J. Micromech. Microeng. 3, 168 (1993).

13. Y.N. Xia et al., Science 273, 347 (1996).

14. Y.N. Xia and G.M. Whitesides, Angew. Chem.Int .E d. Engl. 37, 550 (1998).

15. C.S. Effenhauser, G.J.M. Bruin, A. Paulus, M. Ehrat, Anal. Chem 69, 3451 (1997).

16. E. Delamarche, A. Bernard, H. Schmid, B. Michel, H. Biebuyck, Science 276, 779 (1997).

17. A.Y. Fu, C. Spence, A. Scherer, F.H. Arnold, S.R. Quake, Nature Biotechnol. 17, 1109 (1999).

18. K. Hosokawa, T. Fujii, I. Endo, Anal. Chem. 71, 4781 (1999).

19. D.C. Duffy, O.J.A. Schueller, S.T. Brittain, G.M. Whitesides, J. Micromech. Microeng. 9,211 (1999)

20. M.A. Unger, H. Chou, T. Thorsen, A. Scherer, S. Quake, Science 288, $113(2000)$.

21. H. Chou, C. Spence, A. Scherer, S. Quake, Proc. Natl. Acad. Sci. USA 96, 11 (1999). 